\title{
Lysine methylation of promoter-bound transcription factors and relevance to cancer
}

\author{
George R Stark ${ }^{1,2}$, Yuxin Wang ${ }^{1,2}$, Tao $\mathrm{Lu}^{1}$ \\ ${ }^{I}$ Department of Molecular Genetics, Lerner Research Institute, Cleveland Clinic, 9500 Euclid Avenue, Cleveland, OH 44195, USA; \\ ${ }^{2}$ Institute of Cancer Biology and Drug Screening, School of Life Science, Lanzhou University, Lanzhou 730000, China
}

p53, NFкB, STAT3, and several other transcription factors are reversibly methylated on lysine residues by enzymes that also modify histones. The methylations of NFKB and STAT3 take place when they are bound to promoters, suggesting a more general model in which the binding of inducible transcription factors to DNA helps to recruit chromatin-modification machinery, which then may modify not only histones but also the bound transcription factors. Mutations of some histone-lysine methyltransferases and demethylases are linked to cancer, and these mutations may alter the methylation not only of histones but also of transcription factors, and thus may be tumorigenic through more than one mechanism.

Keywords: posttranslational modification; histones; transcription factors

Cell Research (2011) 21:375-380. doi:10.1038/cr.2010.174; published online 14 December 2010

In recent years, much attention has been focused on posttranslational modification of chromatin because of its critical role in regulating gene expression [1]. The $\mathrm{N}$-terminal tails of histones, as well as positions in the globular domains, carry methylations, acetylations, phosphorylations, ADP ribosylations, ubiquitinations, sumoylations, and other modifications [2]. Many different amino acid residues of each of the four core histones have been identified as modification sites [3] and some lysine side chains can be methylated or acetylated alternatively. These modifications provide entry sites for accessory proteins that help to determine higher order chromatin organization, leading to the activation or inactivation of specific genes. The lysine $\varepsilon$-amino groups can receive one, two, or three methyl groups and the extent of lysine methylation at a single lysine residue can be read differently by different effector proteins [4]. H3K4 methylation is associated with an early-elongating form of RNA polymerase II at actively transcribed genes, H3K9 and H3K27 methylation are linked to heterochromatin formation, while $\mathrm{H} 3 \mathrm{~K} 36$ methylation provides a stable molecular mechanism for establishing chromatin

Correspondence: George R Stark

Tel: 216-444-6062; Fax: 216-444-0512

E-mail: starkg@ccf.org context throughout the genome by distinguishing potential regulatory regions from transcribed chromatin [5]. Moreover, increasing evidence indicates that nonhistone proteins are subject to reversible acetylation or methylation by histone-modifying enzymes. Among these nonhistone targets are transcription factors, hormone receptors, signal transducers, chaperones, and proteins of the cytoskeleton [6-12]. Although the acetylation of nonhistone proteins has been appreciated for some time $[9,13,14]$, their methylation has been recognized only more recently. Here, we provide a summary of recent work showing lysine methylation of transcription factors that have well-recognized roles in tumorigenesis and of the effects of mutations of lysine methyltransferases and demethylases in cancer, and we cite evidence that at least some modifications take place only on promoter-bound transcription factors.

\section{Lysine methylation of nonhistone proteins}

Reversible modification of histones by methylation and demethylation, which occur at both lysine and arginine residues, plays an important role in many biological processes, including transcriptional regulation, heterochromatin formation, $\mathrm{X}$-inactivation, and genomic imprinting [15-17]. Recent work shows that p53, NFKB, STAT3, and other nonhistone proteins (see below) are 
also methylated reversibly on lysine residues through the actions of enzymes previously known to modify only histones. For p53, as summarized in a recent review [18], the reactions occur on K370, K372, and K382, with consequences for function that depend on the site and the degree of methylation. K370 is monomethylated by the H3K4 methylase SMYD2, leading to repression of transcription [19], and is dimethylated at this site by an unknown methylase. Dimethylation of K370 provides a binding site for the coactivator 53BP1 and thus is strongly activated. K370me and K370me2 are both demethylated by the H3K4 demethylase LSD1 [20]. K372 is methylated by the H3K4 methylase SET7/9, which enhances subsequent acetylation of this residue, in turn stabilizing p53 [21]. Shi et al. [22] reported that SET8 monomethylates p53 on K382, with suppression of activity on some target genes but little effect on others. Similar gene-specific effects of dimethylating STAT3 have been noted by Yang et al. [8].

Our laboratory has found that, in response to an activating signal such as treatment with IL-1, the p65 subunit of $\mathrm{NF}_{\mathrm{K}} \mathrm{B}$ is inducibly methylated and demethylated on two specific lysine residues by chromatin-remodeling enzymes in ways that profoundly affect its function [7]. The activating monomethylation of K218 and dimethylation of K221 are both catalyzed by an H3K36 methylase, nuclear receptor-binding SET domain-containing protein 1 (NSD1); and these methyl groups are removed by an H3K36 demethylase, F-box leucine repeat rich protein 11 (FBXL11), leading to inactivation of NFKB. Remarkably, the expression of the FBXL11 gene is induced in response to $\mathrm{NF}_{\kappa} \mathrm{B}$ activation, forming a novel negative feedback loop similar to the one that involves the wellknown negative regulator $\mathrm{I}_{\kappa} \mathrm{B}$ [23]. Yang et al. [24] reported that $\mathrm{K} 314$ and $\mathrm{K} 315$ of p65 are monomethylated by SET7/9 in response to $\mathrm{NF}_{\kappa} \mathrm{B}$ activation, an inhibitory modification that stimulates proteosome-mediated degradation of promoter-associated p65. Ea et al. [25] reported that SET7/9 specifically methylates p65 at K37, and this methylation is restricted to the nucleus and regulates the promoter binding of p65. The methylation of $\mathrm{p} 65$ at K37 affects the stability of DNA-p65 complexes, which in turn regulates the recruitment of p65 to the promoter and the induction of a subset of $\mathrm{NF}_{\kappa} \mathrm{B}$-regulated genes. Levy et al. [26] showed that SETD6 monomethylates p65 on $\mathrm{K} 310$, leading to the induction of a repressed state at $\mathrm{NF}_{\kappa \mathrm{B}}$ target genes through the binding of G9a-like protein (GLP). Phosphorylation of S311 blocks GLP binding, and thus drives target gene expression. Correlations between serine phosphorylation and lysine acetylation were reported by Chen et al. [27], who found that mutation of S276 or S536 of p65 sharply inhibited the acetylation of K310.
In response to IL-6, the methylation of K140 of STAT3 is catalyzed by the H3K4 methylase SET7/9, and the methyl groups are removed by the H3K4 demethylase LSD1 [8]. As is also true for $\mathrm{NF}_{\kappa} \mathrm{B}$, the association of STAT3 with the modifying enzymes is signal dependent. Methylation blocks the binding of STAT3 to a DNA probe and prevention of methylation by K140A or K140R mutation greatly enhances the induction of a subset of genes that respond to IL-6.

Several additional examples of transcription factors and chromatin regulatory proteins that are methylated by histone-modifying enzymes have come to light recently. Many of these proteins are methylated by SET7/9. TAF10, a subunit of the basal eukaryotic transcription factor TFIID, is monomethylated by SET7/9 at K189 [28], increasing its affinity for RNA polymerase II and specific target gene expression. TAF7, another subunit of TFIID, is also monomethylated by SET7/9 at K5 [29]. The estrogen receptor $\alpha$, a member of a large conserved super-family of steroid hormone nuclear receptors that regulates many physiological pathways by acting as a ligand-dependent transcription factor, is monomethylated by SET7/9 at K302, resulting in receptor accumulation and stabilization in the nucleus and target gene expression [30]. Another nuclear hormone receptor, the androgen receptor (AR), is methylated on K632 by SET7/9 [31]. This methylation is necessary for enhancing the transcriptional activity of AR by facilitating both interdomain communication between the $\mathrm{N}$ - and $\mathrm{C}$-termini and recruitment to androgen-target genes. Recently, Kontaki et al. [32] demonstrated that SET7/9 methylated E2F1 at K185, which prevented E2F1 accumulation during DNA damage and activation of its pro-apoptotic target gene $p 73$. Similar to STAT3, the methyl group of K185-methylated E2F1 can be removed by LSD1, which is required for E2F1 stabilization and apoptotic function. In addition, methylation of E2F1 at K185 inhibits its acetylation and phosphorylation at distant positions and, in parallel, stimulates ubiquitination and degradation of the protein [32]. The P300/CBP-associated factor (PCAF) is an acetyltransferase that has been implicated in many cellular processes. Recently, PCAF was found to be methylated at K78 and K89 by SET7/9 [33]. This phenomenon suggests that a histone modifying enzyme can also be modified by lysine methylation. The retinoblastoma tumor suppressor protein $(\mathrm{Rb})$ is monomethylated at K873 by SET7/9, which is required for Rb-dependent cell cycle arrest, transcriptional repression, and $\mathrm{Rb}-$ dependent differentiation as well as interaction with the heterochromatin protein HP1 [10]. The Rb protein is also shown to be methylated at K860 by SMYD2, the same histone H3K4 methyltransferase that methylates p53 [19, 34]. Methylation of K860 of Rb provides a direct bind- 
ing site for the methyl-binding domain of the transcriptional repressor L3MBTL1 [1(3)mbt-like 1], which helps to regulate the biological function of $\mathrm{Rb}$ in mammalian cells.

G9a, another histone-lysine methyltransferase, catalyzes mono-, di-, and tri- methylation of histone $\mathrm{H} 3 \mathrm{~K} 9$ $[35,36]$. Knockout of G9a results in a decrease in global $\mathrm{H} 3 \mathrm{~K} 9 \mathrm{me}$ and $\mathrm{H} 3 \mathrm{~K} 9 \mathrm{me} 2$ levels in vivo [36]. In vitro, G9a catalyzes the formation of $\mathrm{H} 3 \mathrm{~K} 9 \mathrm{me}$ and $\mathrm{H} 3 \mathrm{~K} 9 \mathrm{me} 2$, as well as $\mathrm{H} 3 \mathrm{~K} 9 \mathrm{me} 3$ after a long incubation [36]. Additionally, G9a also methylates H3K27 in vitro [36]. Several nonhistone proteins can be methylated by G9a. The CCAAT/enhancer-binding protein- $\beta(\mathrm{C} / \mathrm{EBP} \beta)$ is methylated at $\mathrm{K} 39$, which may create a binding site for a repressive protein complex or enhance interaction with C/EBP $\beta$ by "reading" methylated K39 [12]. Lee et al. [37] reported that reptin, a chromatin-remodeling factor, is methylated at $\mathrm{K} 67$ under hypoxic conditions by G9a. Methylated reptin binds to the promoters of a subset of hypoxia-responsive genes and downregulates transcription of genes involved in metabolism and tumor development, to modulating cellular responses to hypoxia. Other nonhistone proteins methylated by G9a include chromodomain Y-like protein, widely interspaced zinc finger motifs protein, and Cockayne syndrome group B protein [38]. Interestingly, G9a is auto-methylated at its $\mathrm{N}$-terminus [38, 39]. The methylation events of these nonhistone targets catalyzed by G9a create binding sites for heterochromatin binding protein HP1, which is likely to have further downstream impact on their cellular functions [39]. Additionally, some nonhistone proteins are methylated by unknown enzymes. For example, the retinoic acid receptor $\alpha(\operatorname{RAR} \alpha)$ can be monomethylated at K109 and K171 and tri-methylated at K347 in response to retinoic acid $[35,36]$. However, the methylases have not yet been identified. Methylation of RAR $\alpha$ plays diverse functions: K-to-R mutations of K109 in the DNA-binding domain results in insensitivity to activation by retinoic acid and impairment of RXR/RAR heterodimer-dependent target gene induction; trimethylation of K347 in the RAR $\alpha$ ligand-binding domain facilities RAR $\alpha$ activation and target gene induction by retinoic acid. With the rapidly increasing number of examples of lysine methylation of nonhistone proteins and the diverse roles that methylation plays, one can appreciate the importance of this modification, both in normal physiology and in the etiology of disease.

\section{Methylation of NFKB and STAT3 takes place after they bind to DNA}

Where are the transcription factors when they are modified? Three studies of $\mathrm{NF} \kappa \mathrm{B}$ and one of STAT3 showed that methylations of these transcription factors take place on promoters in the context of chromatin. Our study of NFKB methylation showed that the p65 subunit is not associated with histone-modifying enzymes until it is activated [7], suggesting that this event only happens after $\mathrm{NF}_{\kappa} \mathrm{B}$ is released from $\mathrm{I}_{\kappa} \mathrm{B}$. Furthermore, the work of Yang et al. [24] provided important evidence that the methylation of NFKB occurs only when it is in the nucleus and can bind to DNA, as a DNA binding-deficient mutant of p65 was no longer a substrate for methylation by SET7/9. Levy et al. [26] showed that methylation of p65 by SETD6 occurs on the chromatin-associated protein. The result of Yang et al. [8], obtained by using mutants of STAT3, showed that this protein must enter the nucleus and bind to DNA to be methylated. Consistently, STAT3 K140me2 is observed solely in the nucleus, even after lengthy exposure of cells to IL-6. In addition, the well-known IL-6-induced phosphorylation of STAT3 on S727, which lies in the transactivation domain and is required for function, occurs only when the tyrosinephosphorylated protein can enter the nucleus and bind to DNA [8]. This finding is similar to the previous observations [40] that the recruitment of STAT1 to chromatin is required for interferon-induced phosphorylation of the S727 transactivation domain. Furthermore, the methylation of STAT3 K140 is prevented by the S727A mutation, suggesting that phosphorylation precedes methylation [8].

In most of the studies cited above, the levels of the methylases and demethylases have been increased or decreased exogenously, with effects on the in vivo functions of the target transcription factors that are consistent with the effects of the methylations. The endogenous levels of some methylases and demethylases are also subject to change, for example, the gene encoding the demethylase FBXL11 is activated by $\mathrm{NF}_{\kappa} \mathrm{B}$ [7] and the genes encoding the demethylases JMJD1A and JMJD2B are induced by HIF-1 $\alpha$ in response to hypoxia [41]. The latter example and the work of Li et al. [42] exemplify studies in which the levels of histone methylases and demethylases are changed, with biological consequences that have been interpreted in terms of altered histone modification. In the future, it will be important to also consider the biological effects of manipulating the levels of these enzymes in terms of their effects on transcription factors, and perhaps also on other proteins important for gene expression.

\section{Histone-lysine methyltransferases and demethy- lases in cancer}

Lysine methylation of histones and nonhistone pro- 
teins leads to transcriptional activation or repression, depending on which residues are modified and the degree of methylation. In cancer, alterations in the methylation patterns of histone $\mathrm{H} 3 \mathrm{~K} 9, \mathrm{H} 3 \mathrm{~K} 20$, and $\mathrm{H} 3 \mathrm{~K} 27$ are associated with aberrant gene silencing, whereas methylations of $\mathrm{H} 3 \mathrm{~K} 4, \mathrm{H} 3 \mathrm{~K} 36$, and $\mathrm{H} 3 \mathrm{~K} 79$ are associated with transcriptional activation. A comprehensive recent review summarizes the roles of histone methyltransferases in cancer [43]. Here, we add new information to the examples presented by Albert and Helin [43]. Analyses based on the use of high-resolution single-nucleotide polymorphism arrays have provided new perspectives. EZH2 (Enhancer of zeste homolog 2), a well-known histone-lysine methyltransferase strongly linked to cancer, is amplified or overexpressed in human breast, prostate, and melanocytic tumors, and therefore has been proposed to be a strong predictor of tumor progression and metastasis [44-46]. Recently, somatic EZH2 mutations have been identified in myelodysplastic syndrome (MDS), myeloproliferative neoplasms (MPN) and MDS/ MPN overlap disorders [47, 48]. ASXL1 (additional sex comb-like 1), a putative polycomb group protein, is a poorly understood tumor suppressor. However, its mutation seems to play a vital role in the pathogenesis of myeloid disorder $[49,50]$. On the other hand, ASXL1 suppresses the RAR pathway by modulating the activity of LSD1 [51]. Gene identification by inhibition of nonsense-mediated mRNA decay revealed inactivation of the histone-lysine methyltransferase gene SETD2 as a common event in clear cell renal carcinoma [52]. SETD2 is non-redundantly responsible for the trimethylation of histone H3K36, and missense mutations of SETD2 result in loss or decrease of $\mathrm{H} 3 \mathrm{~K} 36 \mathrm{me} 3$ in the development of these tumors. UTX (ubiquitously transcribed X chromosome tetratricopeptide repeat protein), a histone-lysine demethylase, is encoded by one of a limited number of genes on the $\mathrm{X}$ chromosome that escapes inactivation in females [53]. Inactivating somatic mutations of UTX result in deregulation of histone $\mathrm{H} 3$-lysine methylation in multiple tumor types, and introduction of wild-type UTX into cancer cells with pre-existing inactivating UTX mutations results in slowing proliferation and marked transcriptional changes [54]. UTX can also remove $\mathrm{H} 3 \mathrm{~K} 27 \mathrm{me} 3$ to enable Rb-dependent cell cycle arrest [55]. The histone H3K36-lysine methyltransferase NSD1 has been linked to tumorigenesis in prostate cancer and childhood acute myeloid leukemia [43]. Our discovery that NSD1 is capable of activating $\mathrm{NF}_{\kappa} \mathrm{B}$ by methylating $\mathrm{K} 218$ and $\mathrm{K} 221$ of its p65 subunit [7] provides a potential mechanism for how NSD1 might contribute to tumor formation, as constitutive activation of $\mathrm{NF}_{\kappa} \mathrm{B}$ is a hallmark of most cancers. Alterations in the methylation status of p53 and STAT3 through mutation of modifying enzymes would also be expected to be relevant to tumorigenesis as p53 is a major tumor suppressor and STAT3 is activated in several different cancers. We speculate that as yet unknown mutations of additional histonemodifying enzymes will also be discovered to facilitate tumorigenesis, not only through their effects on posttranslational modifications of histones but also through changes in the modifications of transcription factors.

\section{Perspectives and hypothesis}

Taken together, these observations lead to an interrelated set of hypotheses (Figure 1 and [56]). First, some modifications of inducible transcription factors may occur, in concert with histone modifications, only when these factors are bound to specific promoters, where the local chromatin remodeling machinery is active. Second, these modifications profoundly affect the functions of the transcription factors at these promoters, altering their stability, transactivation potency, and affinity for DNA, and thus affecting the strength and duration of inducible gene expression. Third, the modifications are gene-specific, leading to differential effects on individual genes that

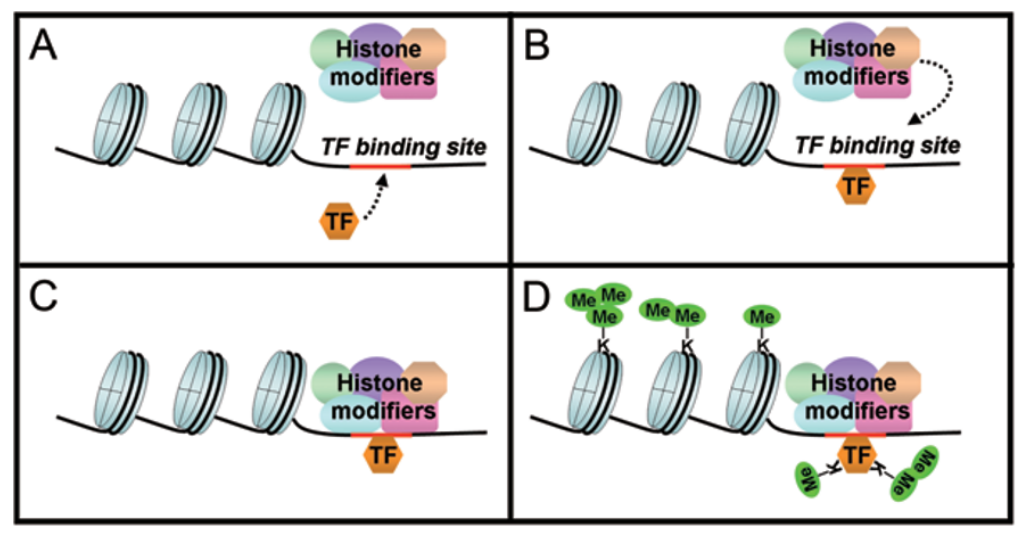

Figure $1 \mathrm{~A}$ model for the kinetics of methylation of chromatin-bound transcription factors by histonemodifying lysine methyltransferases. (A) The transcription factor (TF) and the methyltransferases are free from DNA. (B) The TF binds to its site. (C) The methyltransferases are recruited. (D) The methyltransferases are activated and catalyze methylations of both histones and the TF. Alternatively, some methyltransferases may be pre-associated with some promoters before the TF arrives. Trimethylation of TFs is uncommon, in contrast to the frequent trimethylation of histones. This figure is adapted from Lu and Stark [56]. 
give plasticity to the dependent biological responses. The ability to methylate a specific transcription factor at each different promoter allows the strength and duration of the induced transcriptional response to be regulated individually, rather than having the plasticity of the response constrained by requiring all promoters to respond similarly. A single-modified species of an activated transcription factor can affect specific sub-classes of promoters differentially, but it may also be true that a single-transcription factor may be modified differently in response to different signals. As a likely example, the well-described differential phosphorylation of serine and threonine residues of $\mathrm{NF}_{\kappa} \mathrm{B}$ in response to different signals [57] may constitute a "bar code" that determines differential activation of promoters. Although a variety of mechanisms are surely required for the cell-type specificity of gene activation, the ability to modify transcription factors differently at specific promoters in different types of cells may contribute importantly. Finally, we think it is highly likely that histone-modifying lysine methyltransferases, and perhaps kinases and acetyl transferases as well, will be found to modify as yet unidentified promoter-bound proteins, catalyzing signal-induced posttranslational modifications that remain to be discovered.

\section{Acknowledgments}

This work was supported by NIH Grant P01 CA62220 and Grant 2009 DFA30990 from the Ministry of Science and Technology of China.

\section{References}

1 Wolffe AP. Transcriptional regulation in the context of chromatin structure. Essays Biochem 2001; 37:45-57.

2 Strahl BD, Allis CD. The language of covalent histone modifications. Nature 2000; 403:41-45.

3 Kouzarides T. Chromatin modifications and their function. Cell 2007; 128:693-705.

4 Sims RJ III, Reinberg D. Histone H3 Lys 4 methylation: caught in a bind? Genes Dev 2006; 20:2779-2786.

5 Rao B, Shibata Y, Strahl BD, Lieb JD. Dimethylation of histone $\mathrm{H} 3$ at lysine 36 demarcates regulatory and nonregulatory chromatin genome-wide. Mol Cell Biol 2005; 25:9447-9459.

6 Yang XJ, Seto E. Lysine acetylation: codified crosstalk with other posttranslational modifications. Mol Cell 2008; 31:449461.

$7 \mathrm{Lu}$ T, Jackson MW, Wang B, et al. Regulation of NF-kappaB by NSD1/FBXL11-dependent reversible lysine methylation of p65. Proc Natl Acad Sci USA 2010; 107:46-51.

8 Yang J, Huang J, Dasgupta M, et al. Reversible methylation of promoter-bound STAT3 by histone-modifying enzymes. Proc Natl Acad Sci USA 2010 Nov 23. doi:10.1073/ pnas. 1016147107

9 Singh BN, Zhang G, Hwa YL, et al. Nonhistone protein acetylation as cancer therapy targets. Expert Rev Anticancer Ther 2010; 10:935-954.

10 Munro S, Khaire N, Inche A, Carr S, La Thangue NB. Lysine methylation regulates the $\mathrm{pRb}$ tumour suppressor protein. Oncogene 2010; 29:2357-2367.

11 Kowenz-Leutz E, Pless O, Dittmar G, Knoblich M, Leutz A. Crosstalk between $\mathrm{C} / \mathrm{EBP}$ beta phosphorylation, arginine methylation, and SWI/SNF/Mediator implies an indexing transcription factor code. EMBO J 2010; 29:1105-1115.

12 Pless O, Kowenz-Leutz E, Knoblich M, et al. G9a-mediated lysine methylation alters the function of CCAAT/enhancerbinding protein-beta. J Biol Chem 2008; 283:26357-26363.

13 Bush EW, McKinsey TA. Protein acetylation in the cardiorenal axis: the promise of histone deacetylase inhibitors. Circ Res 2010; 106:272-284.

14 Lu Z, Scott I, Webster BR, Sack MN. The emerging characterization of lysine residue deacetylation on the modulation of mitochondrial function and cardiovascular biology. Circ Res 2009; 105:830-841.

15 Martin C, Zhang Y. The diverse functions of histone lysine methylation. Nat Rev Mol Cell Biol 2005; 6: 838-849.

16 Bedford MT, Richard S. Arginine methylation an emerging regulator of protein function. Mol Cell 2005; 18:263-272.

17 Klose RJ, Kallin EM, Zhang Y. JmjC-domain containing proteins and histone demethylation. Nat Rev Genet 2006; 7:715727.

18 Huang J, Berger SL. The emerging field of dynamic lysine methylation of non-histone proteins. Curr Opin Genet Dev 2008; 18:152-158.

19 Huang J, Perez-Burgos L, Placek BJ, et al. Repression of p53 activity by Smyd2-mediated methylation. Nature 2006; 444:629-632.

20 Huang J, Sengupta R, Espejo AB, et al. p53 is regulated by the lysine demethylase LSD1. Nature 2007; 449:105-108.

21 Ivanov GS, Ivanova T, Kurash J, et al. Methylation-acetylation interplay activates p53 in response to DNA damage. Mol Cell Biol 2007; 27:6756-6769.

22 Shi X, Kachirskaia I, Yamaguchi H, et al. Modulation of p53 function by SET8-mediated methylation at lysine 382. Mol Cell 2007; 27:636-646.

23 Lu T, Jackson MW, Singhi AD, et al. Validation-based insertional mutagenesis to identify the FBXL11 as a negative regulator of NF-kappa B. Proc Natl Acad Sci USA 2009; 106:16339-16344.

24 Yang XD, Huang B, Li M, Lamb A, Kelleher NL, Chen LF. Negative regulation of NF-kappaB action by Set9-mediated lysine methylation of the RelA subunit. EMBO J 2009; 28:1055-1066.

25 Ea CK, Baltimore D. Regulation of NF-kappaB activity through lysine monomethylation of p65. Proc Natl Acad Sci USA 2009; 106:18972-18977.

26 Levy D, Kuo AJ, Chang Y, et al. SETD6 lysine methylation of RelA couples GLP activity at chromation to tonic repression of NF-kappaB signaling. Nat Immunol in press.

27 Chen LF, Williams SA, Mu Y, et al. NF-kappaB RelA phosphorylation regulates RelA acetylation. Mol Cell Biol 2005; 25:7966-7975.

28 Kouskouti A, Scheer E, Staub A, Tora L, Talianidis I. Genespecific modulation of TAF10 function by SET9-mediated 
methylation. Mol Cell 2004; 14:175-182.

29 Couture JF, Collazo E, Hauk G, Trievel RC. Structural basis for the methylation site specificity of SET7/9. Nat Struct Mol Biol 2006; 13:140-146.

30 Subramanian K, Jia D, Kapoor-Vazirani P, et al. Regulation of estrogen receptor alpha by the SET7 lysine methyltransferase. Mol Cell 2008; 30:336-347.

31 Gaughan L, Stockley J, Wang N, et al. Regulation of the androgen receptor by SET9-mediated methylation. Nucleic Acids Res 2010 Oct 19. doi:10.1093/nar/gkq861

32 Kontaki H, Talianidis I. Lysine methylation regulates E2F1induced cell death. Mol Cell 2010; 39:152-160.

33 Masatsugu T, Yamamoto K. Multiple lysine methylation of PCAF by Set 9 methyltransferase. Biochem Biophys Res Commun 2009; 381:22-26.

34 Saddic LA, West LE, Aslanian A, et al. Methylation of the retinoblastoma tumor suppressor by SMYD2. J Biol Chem 2010 Sep 24. doi:10.1074/jbc.M110.137612

35 Huq MD, Tsai NP, Khan SA, Wei LN. Lysine trimethylation of retinoic acid receptor-alpha: a novel means to regulate receptor function. Mol Cell Proteomics 2007; 6:677-688.

36 Huq MD, Ha SG, Wei LN. Modulation of retinoic acid receptor alpha activity by lysine methylation in the DNA binding domain. J Proteome Res 2008; 7:4538-4545.

37 Lee JS, Kim Y, Kim IS, et al. Negative regulation of hypoxic responses via induced Reptin methylation. Mol Cell 2010; 39:71-85.

38 Rathert P, Dhayalan A, Murakami M, et al. Protein lysine methyltransferase G9a acts on non-histone targets. Nat Chem Biol 2008; 4:344-346.

39 Patnaik D, Chin HG, Estève PO, Benner J, Jacobsen SE, Pradhan S. Substrate specificity and kinetic mechanism of mammalian G9a histone H3 methyltransferase. J Biol Chem 2004; 279:53248-53258.

40 Sadzak I, Schiff M, Gattermeier I, et al. Recruitment of Stat1 to chromatin is required for interferon-induced serine phosphorylation of Stat1 transactivation domain. Proc Natl Acad Sci USA 2008; 105:8944-8949.

41 Berger SL. Out of the jaws of death: PRMT5 steers p53. Nat Cell Biol 2008; 10:1389-1390.

42 Li Y, Reddy MA, Miao F, et al. Role of the histone H3 lysine 4 methyltransferase, SET7/9, in the regulation of NF-kappaBdependent inflammatory genes. Relevance to diabetes and inflammation. J Biol Chem 2008; 283:26771-26781.

43 Albert M and Helin K. Histone methylases in cancer. Semin Cell Dev Biol 2010; 21: 209-220.
44 Varambally S, Dhanasekaran SM, Zhou M, et al. The polycomb group protein EZH2 is involved in progression of prostate cancer. Nature 2002; 419:624-629.

45 Bracken AP, Pasini D, Capra M, Prosperini E, Colli E, Helin K. $\mathrm{EZH} 2$ is downstream of the pRb-E2F pathway, essential for proliferation and amplified in cancer. EMBO J 2003; 22:53235335 .

46 Kleer CG, Cao Q, Varambally S, et al. EZH2 is a marker of aggressive breast cancer and promotes neoplastic transformation of breast epithelial cells. Proc Natl Acad Sci USA 2003; 100:11606-11611.

47 Ernst T, Chase AJ, Score J, et al. Inactivating mutations of the histone methyltransferase gene EZH2 in myeloid disorders. Nat Genet 2010; 42:722-726.

48 Nikoloski G., Langemeijer SM, Kuiper RP, et al. Somatic mutations of the histone methyltransferase gene EZH2 in myelodysplastic syndromes. Nat Genet 2010; 42:665-667.

49 Boultwood J, Perry J, Zaman R, et al. High-density single nucleotide polymorphism array analysis and ASXL1 gene mutation screening in chronic myeloid leukemia during disease progression. Leukemia 2010; 24:1139-1145.

50 Carbuccia N, Murati A, Trouplin V, et al. Mutations of ASXL1 gene in myeloproliferative neoplasms. Leukemia 2009; 23:2183-2186.

51 Lee SW, Cho YS, Na JM, et al. ASXL1 represses retinoic acid receptor-mediated transcription through associating with HP1 and LSD1. J Biol Chem 2010; 285:18-29.

52 Duns G, van den Berg E, van Duivenbode I, et al. Histone methyltransferase gene SETD2 is a novel tumor suppressor gene in clear cell renal cell carcinoma. Cancer Res 2010; 70:4287-4291.

53 Greenfield A, Carrel L, Pennisi D, et al. The UTX gene escapes X inactivation in mice and humans. Hum Mol Genet 1998; 7:737-742.

54 van Haaften G, Dalgliesh GL, Davies H, et al. Somatic mutations of the histone H3K27 demethylase gene UTX in human cancer. Nat Genet 2009; 41:521-523.

55 Wang JK, Tsai MC, Poulin G, et al. The histone demethylase UTX enables Rb-dependent cell fate control. Genes Dev 2010; 24:327-332.

$56 \mathrm{Lu}$ T, Stark GR. Use of forward genetics to discover novel regulators of NF-kappa B. Cold Spring Harb Perspect Biol 2010; 2:253-264.

57 O'Shea JM, Perkins ND. Regulation of the RelA (p65) transactivation domain. Biochem Soc Trans 2008; 36:603-608. 